\title{
O desperdício de materiais assistenciais percebido por graduandos de enfermagem
}

\author{
Magaly Cecília Franchini Reichert ${ }^{1}$, Thaís Gomes Lozovoi ${ }^{2}$, Maria D'Innocenzo ${ }^{3}$
}

\footnotetext{
${ }^{1}$ Enfermeira, Mestre em Ciências. Discente do Programa de Pós-Graduação em Enfermagem da Escola Paulista de Enfermagem da Universidade Federal da São Paulo, Nível Doutorado. São Paulo SP, Brasil. E-mail: magaly.reichert@unifesp.br.

${ }^{2}$ Enfermeira. Enfermeira do Hospital São Paulo, Hospital Universitário da Universidade Federal da São Paulo. São Paulo SP, Brasil. E-mail: thaislozovoi@hotmail.com.

${ }^{3}$ Enfermeira, Doutora em Enfermagem. Docente do Programa de Pós-Graduação em Enfermagem da Escola Paulista de Enfermagem da Universidade Federal da São Paulo, Nível Doutorado. São Paulo SP, Brasil. E-mail: mdinnocenzo@unifesp.br.
}

Recebido: 08/07/2016.

Aceito: 03/04/2017.

Publicado: 21/09/2017.

\section{Como citar esse artigo:}

Reichert MCF, Lozovoi TG, D'Innocenzo M. O desperdício de materiais assistenciais percebido por graduandos de enfermagem. Rev. Eletr. Enf. [Internet] 2017 [acesso em:_____ ];19:a27. Disponível em: http://dx.doi.org/10.5216/ree.v19.42243.

\begin{abstract}
RESUMO
O estudo teve como objetivo identificar a opinião dos graduandos de enfermagem sobre o desperdício de materiais assistenciais nas atividades práticas de ensino. Estudo exploratório, descritivo com abordagem quantitativa, cuja amostra foi composta por 186 graduandos que responderam a um instrumento com assertivas medidas pela escala de Likert. Mais da metade dos graduandos acreditaram que as instituições onde realizaram estágio têm desperdício de materiais; $76 \%$ dos graduandos da quarta série $(p<0,001)$ reconheceram desperdiçar materiais durante os estágios e $89 \%$ da mesma série $(p<0,001)$ atribuíram o desperdício à realização de um procedimento pela primeira vez. O estudo possibilitou a discussão do desperdício de materiais durante a graduação em enfermagem, alertando sobre a importância da gestão adequada desses recursos além da responsabilidade da enfermagem com o meio ambiente e práticas sustentáveis. Os achados indicam novas possibilidades para o desenvolvimento do tema e estratégias que podem ser testadas em futuros estudos.
\end{abstract}

Descritores: Educação em Enfermagem; Recursos Materiais em Saúde; Controle de Custos.

\section{INTRODUÇÃO}

Os materiais utilizados na assistência à Saúde são recursos essenciais, que afetam diretamente a qualidade do cuidado prestado, consomem significativamente parte dos recursos financeiros das instituições de saúde representando um gasto de 15 a $25 \%$ do total das despesas nessas instituições ${ }^{(1)}$. Desta forma, os materiais assumem papel de destaque para as organizações de saúde devido ao aumento de custos no setor e aos desafios destas organizações em oferecer serviços com qualidade e custos viáveis. A gestão adequada de materiais reduz custos e diminui desperdícios ${ }^{(2)}$, estratégias importantes neste cenário. 
A Organização Mundial da Saúde (OMS) em seu relatório de 2010, que discorreu sobre o financiamento dos sistemas de saúde e teve como um dos tópicos: "Promover a eficiência e eliminar o desperdício", discutiu a importância de assegurar a utilização dos recursos de modo eficiente, destacando a oportunidade desta ação em todos os países. O relatório também apontou o desperdício e a corrupção como as principais causas de ineficiência dos sistemas de saúde representando entre $20 \%$ e $40 \%$ de todos os gastos em saúde ${ }^{(3)}$.

No Brasil no ano de 2013, o Ministério da Saúde fez uma publicação destinada aos municípios descrevendo as ações para aprimorar o SUS e garantir um atendimento de qualidade aos usuários. Dentre as principais ações temos o combate ao desperdício de recursos e o aprimoramento da gestão ${ }^{(4)}$.

Uma das preocupações atuais no cenário da saúde é a suficiência do sistema para atender todas as demandas da população, sendo a alocação de recursos um ponto crítico na gestão em saúde ${ }^{(5)}$.

No contexto apresentado, o enfermeiro assume papel de destaque na gestão de materiais assistenciais, pois coordena a assistência de enfermagem determinando o material necessário para sua execução e, desta forma, está diretamente envolvido na tomada de decisão sobre esses recursos ${ }^{(6)}$. 0 enfermeiro racionaliza os recursos materiais disponíveis, pois avalia as necessidades dos pacientes e equipe de enfermagem, visando a segurança e qualidade do cuidado ${ }^{(7)}$.

Apesar da importante atuação do enfermeiro, quatro estudos sobre desperdício realizados em três hospitais brasileiros e um hospital nos Estados Unidos da América apontaram os materiais como um dos itens mais desperdiçados ${ }^{(8-11)}$.

A enfermagem tem grande responsabilidade sobre a utilização dos materiais assistenciais com consequências de igual magnitude, seja em relação à qualidade da assistência, aos resultados financeiros ou ao impacto causado ao meio ambiente.

Considerando que o aumento do lixo gerado pela assistência à saúde, principalmente quando não tratado adequadamente, traz consequências diretas à qualidade de vida da população uma vez que impacta em todo meio ambiente ${ }^{(12)}$, a reflexão sobre o desperdício de materiais é um exercício urgente que deve ser iniciado na formação dos enfermeiros para que possam atuar em consonância com a realidade onde estão inseridos, promovendo mudanças que beneficiem todos os usuários do SUS e a população em geral.

Estudos mostraram lacunas na formação dos futuros enfermeiros referentes ao conhecimento sobre o custo dos materiais utilizados na assistência ${ }^{(13)}$ e o quanto esse conhecimento pode influenciar no desperdício desses insumos ${ }^{(14)}$. Assim, um estudo com aos graduandos de enfermagem sobre o desperdício de materiais poderá contribuir para a reflexão sobre o uso adequado desses insumos, promovendo a conscientização sobre o desenvolvimento sustentável e subsidiando o ensino dos futuros enfermeiros.

O presente estudo teve os seguintes objetivos: identificar a opinião dos graduandos de enfermagem sobre o desperdício de materiais assistenciais em relação à sua eventual ocorrência nas atividades práticas de ensino; identificar os conteúdos transmitidos pelos docentes e possíveis fatores causadores do desperdício; descrever os materiais desperdiçados e as sugestões dos graduandos para sua redução. 


\section{MÉTODO}

Estudo descritivo, exploratório com abordagem quantitativa. Foi realizado na Escola Paulista Enfermagem (EPE) - Universidade Federal de São Paulo. O curso de enfermagem da EPE acontece em horário integral, tem entrada anual de 88 graduandos, é dividido em quatro séries com regime anual e tempo de integralização mínimo de quatro anos. As atividades práticas do curso são realizadas em diversos cenários, que dependem da especificidade dos conteúdos a serem abordados: laboratórios, unidades de internação hospitalar e equipamentos de atenção primária de saúde. Os estágios têm início na primeira série com carga horária menor que vai aumentando gradativamente de acordo com a progressão do aluno no curso. A terceira e quarta séries se destacam com os maiores percentuais de horas de estágio sendo respectivamente $53 \%$ e $85 \%$, já a primeira e segunda séries possuem percentuais menores $(14 \% \text { e } 39 \%)^{(15)}$. Em alguns estágios os graduandos vivenciam situações reais de assistência à saúde tendo contato com a equipe de enfermagem, pacientes e demais profissionais.

A população do estudo foi constituída por graduandos de enfermagem regularmente matriculados na segunda, terceira e quarta séries. Foram excluídos os graduandos da primeira série, pois no período da coleta de dados não haviam concluído a Unidade Curricular onde iniciam a realização de procedimentos específicos para o desenvolvimento de habilidades técnicas de enfermagem com utilização de materiais assistenciais. Foram incluídos os graduandos das demais séries (2a $3^{a}$ e e $4^{a}$ ) que estavam presentes nas datas da coleta de dados. A amostra por conveniência totalizou 186 graduandos.

Os graduandos foram convidados a participar do estudo em sala de aula antes do início das aulas ou ao término das mesmas em datas aleatórias, sendo os dados coletados entre maio e junho de 2014. O termo de consentimento livre e esclarecido foi assinado por aqueles graduandos que concordaram em participar do estudo após terem sido informados dos objetivos e da condução da pesquisa pelas pesquisadoras que aguardaram no local o preenchimento do instrumento.

O instrumento de coleta de dados foi construído com base na revisão de literatura sobre o desperdício de materiais, na experiência das autoras e submetido à análise de duas enfermeiras professoras doutoras, com experiência em recursos financeiros e materiais. A concordância entre as duas avaliadoras foi de $88 \%$ e as sugestões foram acatadas e modificadas no instrumento. Um pré-teste foi realizado com 11 graduandos a fim de se assegurar a compreensão, identificação de possíveis dificuldades no preenchimento e sua representatividade de situações vivenciadas pelos estudantes nos campos de prática. Os graduandos que participaram do pré-teste não fizeram parte da amostra.

O instrumento resultou em questões abertas sobre: a caracterização dos participantes, os materiais desperdiçados nas atividades práticas nos campos de estágio e sugestões para redução do desperdício. Também contou com 10 assertivas que foram medidas por meio de uma escala de Likert abordando a opinião dos graduandos sobre o desperdício de materiais, conteúdos ministrados e suas possíveis causas.

Neste estudo entendem-se como materiais assistenciais utilizados durante as atividades práticas de ensino aqueles itens descartáveis, utilizados para realizar os procedimentos de enfermagem, consumidos no 
momento em que a assistência foi prestada pelo graduando e que foram fornecidos pelos serviços de saúde onde o estágio foi realizado.

As variáveis do estudo foram categorizadas, codificadas e armazenadas em planilha Microsoft Excel ${ }^{\circ}$. Para as comparações foi aplicado o teste Exato de Fisher, Qui-Quadrado ou o Qui-Quadrado de Máxima Verossimilhança, quando apropriado. As variáveis quantitativas foram avaliadas quanto a sua distribuição normal pelo teste de Shapiro-Wilk, e foram expressas em termos de mediana e primeiro e terceiro quartis (Q1 e Q3). Em todos os testes aplicados, os resultados foram significativos quando o nível descritivo (p-valor) foi inferior a 0.05. Todos os testes foram realizados com o auxílio do programa SAS for Windows, versão 9.2 (SAS Institute Inc., Cary, NC, USA.) com apoio de um estatístico. As variáveis categóricas foram analisadas descritivamente.

O estudo seguiu os preceitos éticos necessários na condução de pesquisas com seres humanos e foi aprovado pelo Comitê de Ética e Pesquisa da Universidade Federal de São Paulo, CAAE: 24410014.4.0000.5505.

\section{RESULTADOS}

Do total de 238 graduandos matriculados nas séries investigadas, obtivemos a participação de 186 (78,2\%), com a seguinte distribuição: $38,7 \%$ da quarta série, $33,3 \%$ da segunda e $28,0 \%$ da terceira. Sendo 93,5\% do sexo feminino, com idade mediana de 21 anos ( $Q 1-20$ anos e Q3 - 23 anos), 70.4\% nunca haviam trabalhado e $90.8 \%$ estavam cursando a primeira graduação. A adesão dos graduandos às assertivas do estudo foi de $99,5 \%$.

Na opinião dos graduandos, aproximadamente metade $(53,2 \%)$ acreditavam que as instituições que recebem graduandos de enfermagem apresentam desperdício maior de materiais ( $p-0,0144), 68,2 \%$ afirmaram se preocupar com as instituições pelos gastos provenientes do desperdício de materiais durante os estágios da graduação e $76 \%$ dos graduandos da quarta série $(p<0,001)$ admitiram desperdiçar materiais nas atividades práticas durante os estágios da graduação.

Com relação aos conteúdos transmitidos, 56,9\% alegaram que durante os estágios os professores ensinaram a importância do uso racional dos materiais e $44,6 \%$, que o desperdício foi pouco abordado em aulas teóricas. A quase totalidade dos graduandos $(96,7 \%)$ compreendeu que o desperdício de materiais impacta nos gastos das instituições de saúde (Figura 1). Parte dos graduandos (47,3\%) referiu ter recebido orientações para um planejamento adequado dos materiais que seriam utilizados nos procedimentos evitando o desperdício. 


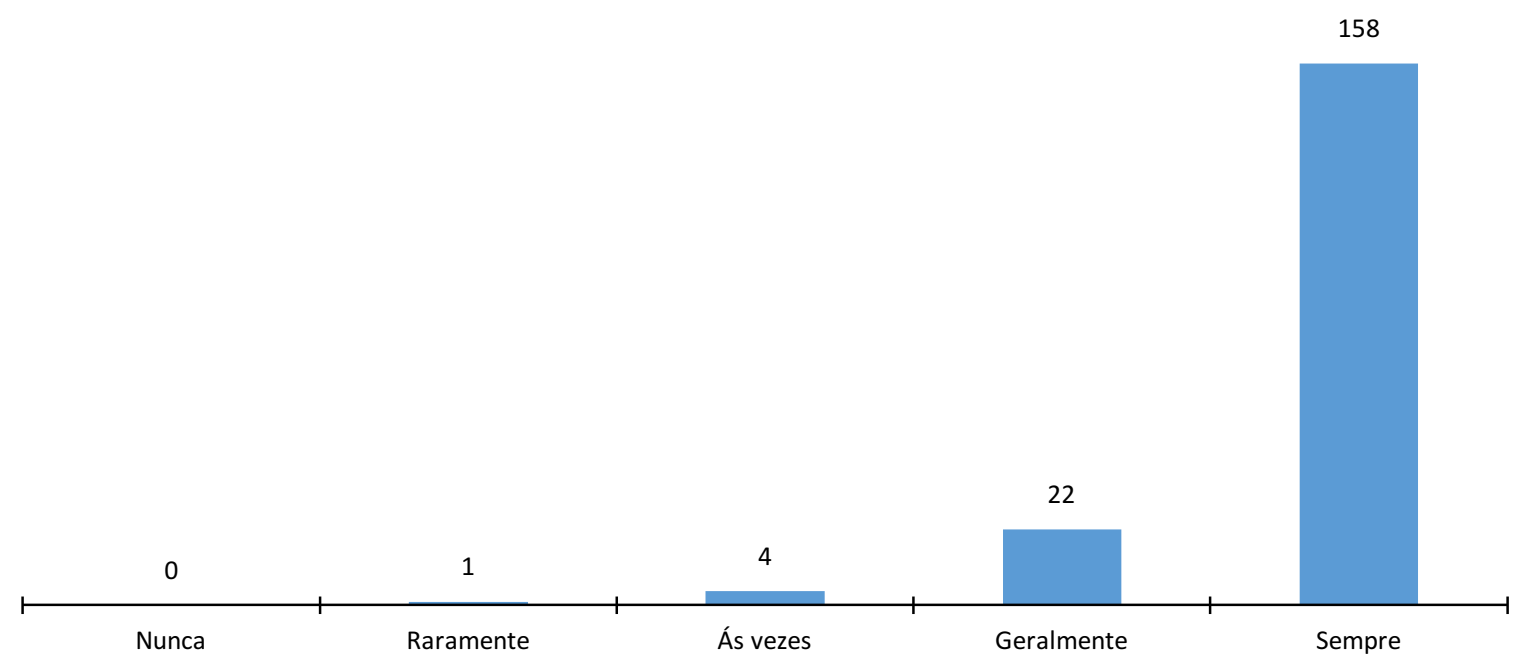

Figura 1: Opinião dos graduandos sobre a assertiva:

“O desperdício de materiais impacta nos gastos das instituições de saúde”. São Paulo, SP, Brasil, 2014.

Os resultados referentes às possíveis causas do desperdício de materiais nos estágios mostraram que a presença do professor causando algum tipo de tensão ou influência constrangedora nunca ou raramente foi observada por $46,8 \%$ dos graduandos. 0 fato de realizar um procedimento pela primeira vez foi apontado como causa de desperdício por $41,3 \%$ dos graduandos com diferença significativa para a quarta série $(\mathrm{p}<$ 0,001 ) onde $89 \%$ concordaram com a assertiva. A variedade de materiais disponíveis nos campos de prática não foi apontada como um possível fator de desperdício por 83,3\%, conforme Figura 2.

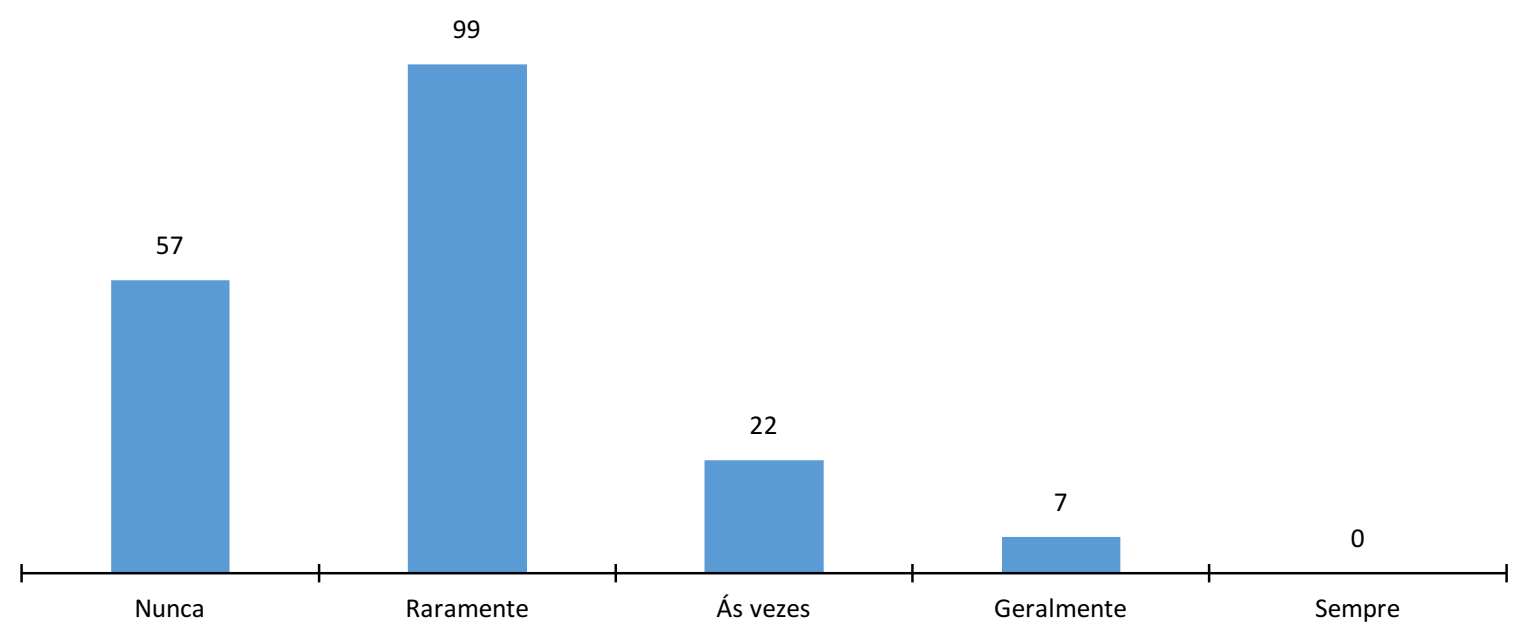

Figura 2: Opinião dos graduandos sobre a assertiva: "Desperdiço materiais nos estágios da graduação devido à variedade dos mesmos". São Paulo, SP, Brasil, 2014.

Ao analisar os materiais desperdiçados durante os estágios da graduação (Tabela 1), observa-se que os principais foram: gaze $(21,7 \%)$, seringa $(19,6 \%)$, agulha $(19,0 \%)$ e luvas $(17,1 \%)$, com pequenas diferenças de frequências entre as séries. Esses itens totalizaram a maioria $(77,4 \%)$ dos materiais citados pelos graduandos. 
Tabela 1: Materiais desperdiçados durante os estágios da graduação segundo opinião dos graduandos. São Paulo, SP, Brasil, 2014.

\begin{tabular}{|c|c|c|c|c|c|c|c|c|}
\hline \multirow{2}{*}{ Materiais } & \multicolumn{2}{|c|}{$2^{\circ}$ Série } & \multicolumn{2}{|c|}{$3^{\circ}$ Série } & \multicolumn{2}{|c|}{$4^{\circ}$ Série } & \multicolumn{2}{|c|}{ Total } \\
\hline & $\mathbf{n}$ & $\%$ & $\mathbf{n}$ & $\%$ & $\mathbf{n}$ & $\%$ & $\mathbf{n}$ & $\%$ \\
\hline Gaze & 32 & 21,5 & 38 & 23,9 & 57 & 20,6 & 127 & 21,7 \\
\hline Seringa & 23 & 15,4 & 34 & 21,4 & 58 & 20,9 & 115 & 19,6 \\
\hline Agulha & 23 & 15,4 & 32 & 20,1 & 56 & 20,2 & 111 & 19,0 \\
\hline Luvas & 31 & 21,0 & 25 & 15,7 & 44 & 15,9 & 100 & 17,1 \\
\hline Algodão & 15 & 10,0 & 14 & 8,8 & 8 & 2,9 & 37 & 6,3 \\
\hline Dispositivo intravenoso & 7 & 4,7 & - & - & 23 & 8,3 & 30 & 5,1 \\
\hline Fita adesiva hipoalergênica & 5 & 3,3 & 10 & 6,3 & 6 & 2,1 & 21 & 3,6 \\
\hline Álcool 70\% & 9 & 6,0 & - & - & 8 & 2,9 & 17 & 3,0 \\
\hline Equipo de soro & - & - & - & - & 11 & 4,0 & 11 & 1,9 \\
\hline Esparadrapo & - & - & 6 & 3,8 & 3 & 1,1 & 9 & 1,5 \\
\hline Outros & 4 & 2,7 & - & - & 3 & 1,1 & 7 & 1,2 \\
\hline Total & 149 & 100 & 159 & 100 & 277 & 100 & 585 & 100 \\
\hline
\end{tabular}

As sugestões para reduzir o desperdício de materiais durante os estágios da graduação totalizaram 171 citações, sendo $86(50,3 \%)$ da quarta série, $43(25,1 \%)$ da segunda e $42(24,6 \%)$ da terceira série. As principais sugestões foram: maior abordagem sobre o desperdício de materiais tanto na teoria quanto na prática (43,3\%); separar a quantidade adequada de materiais para a realização dos procedimentos $(30,4 \%)$ e conscientização dos graduandos quanto ao custo dos materiais $(9,4 \%)$, conforme tabela 2 .

Tabela 2: Sugestões dos graduandos para redução do desperdício de materiais durante os estágios da graduação. São Paulo, SP, Brasil, 2014.

\begin{tabular}{ccc}
\hline Sugestões & $\mathbf{n}$ & $\%$ \\
\hline Maior abordagem sobre o desperdício de materiais na teoria e prática. & 74 & 43,3 \\
Separar a quantidade adequada de materiais para realização dos procedimentos. & 52 & 30,4 \\
Conscientizar os graduandos quanto ao custo dos materiais e desperdício. & 16 & 9,4 \\
Menor pressão do professor durante as atividades práticas. & 15 & 8,8 \\
Maior treinamento no laboratório de simulação realística. & 10 & 5,8 \\
Outros & 4 & 2,3 \\
\hline Total & $\mathbf{1 7 1}$ & $\mathbf{1 0 0}$ \\
\hline
\end{tabular}

Apesar do expressivo número $(n=131)$ de graduandos que nunca haviam trabalhado, foram comparadas as assertivas segundo o grupo de graduandos que trabalharam e os que não trabalharam, porém nenhuma evidência significativa desta diferença foi identificada entre esses grupos. Devido ao fato de mais de $90 \%$ dos graduandos estarem cursando a sua primeira graduação, não se realizou nenhum teste comparativo para esse agrupamento.

\section{DISCUSSÃO}

Os resultados evidenciaram discrepâncias entre as opiniões dos graduandos da quarta série em relação aos demais. Este grupo também apresentou resultados significativos em relação à maior percepção que têm do próprio desperdício de materiais durante as atividades práticas na graduação. Também foi a série que fez mais sugestões para evitar o desperdício. É esperado que os graduandos na quarta série, no processo de construção do conhecimento e desenvolvimento de competências, apresentem uma visão ampla e crítica, que contextualize o futuro profissional, devido a maior carga horária em campos de prática, assim como 
subsídios teóricos ${ }^{(16)}$.

O estudo mostrou o reconhecimento por parte dos graduandos de um desperdício maior de materiais nas instituições que recebem estudantes de enfermagem para atividades práticas e o impacto financeiro deste desperdício às mesmas. Esse resultado corrobora com os estudos que apontam os hospitais de ensino como estruturas caras e que apresentam custos mais elevados que os demais, sendo uma das causas as atividades desenvolvidas para formação de recursos humanos na área da saúde ${ }^{(17)}$.

Os graduandos referiram que parte dos docentes destacou a importância de usar os recursos materiais de maneira adequada, sem excessos, durante as atividades práticas, fato que pode ser confirmado pelas respostas, onde se mostraram preocupados com os gastos que as instituições de saúde podem ter quando o desperdício de materiais é uma prática entre os estagiários de enfermagem. Essa opinião dos alunos é condizente com o momento atual, onde as organizações de saúde necessitam aprimorar o controle de custos e o uso eficiente de seus recursos para sua sobrevivência ${ }^{(16-17)}$.

Quanto aos conteúdos ministrados sobre o desperdício, os resultados mostraram a necessidade de uma reflexão sobre o planejamento dos materiais em relação à adequação da quantidade de materiais separados para a realização dos procedimentos de enfermagem. Tal fato pode ter como base a deficiência na formação acadêmica sobre os aspectos ambientais ${ }^{(18)}$ propiciando o uso indiscriminado dos materiais assistenciais. Estudo ${ }^{(19)}$ realizado em cinco cursos de graduação em enfermagem no sul do Brasil mostrou a importância dos docentes estarem sensibilizados com as demandas socioambientais, pois só assim poderão despertar nos futuros enfermeiros valores de responsabilidade com o meio ambiente e com a sociedade.

Os graduandos apontaram a necessidade de um reforço sobre o tema desperdício em aulas teóricas e nas atividades práticas. Vale destacar que os professores têm um papel fundamental na prevenção do desperdício, pois é a partir deles que os graduandos serão orientados. Os professores atuam como exemplo para os graduandos, para isto é fundamental que os mesmos se conscientizem quanto ao papel de destaque que a enfermagem tem na gestão de custos $^{(17,20-21)}$, além da responsabilidade que têm por sua capacitação para o exercício competente de suas ações ${ }^{(22)}$.

Uma das principais causas para o desperdício dos materiais foi a falta de experiência dos graduandos. Estudo sobre o desperdício relacionado aos materiais também apontou a inexperiência e a pouca habilidade de estudantes presentes na unidade de internação como uma das causas do desperdício ${ }^{(8)}$.

Os materiais desperdiçados mais citados foram gaze, seringa, agulha e luvas. Estes resultados coincidem com dois estudos realizados em hospitais universitários onde as luvas, gaze e seringa foram também os materiais mais desperdiçados ${ }^{(8-9)}$. Em outro estudo realizado em uma Unidade de Terapia Intensiva Neonatal, a gaze e a luva foram os materiais mais desperdiçados ${ }^{(10)}$.

Assim podemos afirmar que esses materiais mais citados pelos graduandos no presente estudo são muito utilizados durante os estágios para o desenvolvimento de habilidades técnicas de enfermagem como preparo e administração de medicações, punção venosa, curativos, além do uso de luvas como equipamento de proteção individual. Estudo mostrou que os materiais utilizados na assistência representaram 31,3\% da 
classe de materiais com maior custo total de um hospital universitário ${ }^{(23)}$, reforçando a importância da atuação do enfermeiro e sua equipe para diminuição do desperdício.

Em relação às sugestões dos graduandos, além das já mencionadas, a conscientização sobre o custo dos materiais foi apontada por 9,4\%. O conhecimento prévio sobre o custo de materiais pode ser uma maneira de prevenir o desperdício segundo opinião de enfermeiros, técnicos e auxiliares de enfermagem, docentes e graduandos de enfermagem ${ }^{(14)}$, assim como um treinamento introdutório sobre o tema antes do início das atividades práticas no hospital ${ }^{(11)}$.

O conhecimento sobre custos é fundamental para que o enfermeiro possa responder aos desafios e demandas na gestão econômica das organizações de saúde ${ }^{(21)}$. Considerando o baixo conhecimento por parte dos graduandos sobre os custos dos materiais ${ }^{(13)}$, a orientação pelos docentes quanto ao custo dos materiais no momento da sua utilização é uma prática que deve ser rapidamente introduzida.

Um treinamento maior de habilidades técnicas em laboratório de simulação realística foi outra sugestão dos graduandos, que apesar de ter sido apontada por um grupo pequeno merece uma análise criteriosa. A utilização da simulação realística tem mostrado resultados positivos no ensino da Enfermagem. Está sendo amplamente utilizada, pois as experiências práticas com os pacientes muitas vezes são limitadas e as oportunidades não são suficientes devido ao número de alunos e a quantidade de habilidades a serem desenvolvidas ${ }^{(24)}$. Estudo sobre a simulação realística durante o estágio de graduandos de enfermagem em cenário hospitalar mostrou-se efetivo na opinião dos estudantes de enfermagem para aperfeiçoar os conhecimentos e segurança ${ }^{(25)}$. O reforço na utilização de simulação de situações práticas para aprimorar as habilidades técnicas dos futuros enfermeiros pode ser uma estratégia para redução do desperdício de materiais, além de melhorar a qualidade e segurança da assistência prestada.

\section{CONCLUSÃO}

Este estudo evidenciou que os graduandos de enfermagem desperdiçam materiais no desenvolvimento das atividades práticas durante a graduação, sendo a principal causa a pouca habilidade técnica para realização dos procedimentos. A investigação permitiu ainda a discussão acerca da importância da eficiência na gestão dos materiais para os futuros enfermeiros com ênfase na redução do desperdício e a conscientização sobre práticas sustentáveis tanto do ponto de vista financeiro quanto ambiental.

O estudo também destacou a atuação do docente na formação dos graduandos sob a ótica da otimização dos materiais sugerindo a necessidade da contextualização teórica sobre os custos dos materiais principalmente durante o desenvolvimento das atividades práticas, assim como a utilização de novas estratégias de ensino que possibilitem maior destreza a esses graduandos na realização da prática assistencial.

A pesquisa foi uma aproximação inicial da temática desperdício de materiais na formação do enfermeiro, com resultados que suscitam novos estudos para ampliação do tema tão importante para a prática da enfermagem. Apresenta limitações, pois analisou as opiniões dos graduandos de enfermagem de 
apenas uma instituição de ensino superior, sendo que resultados diferentes podem ser obtidos se aplicada a outras realidades.

\section{REFERÊNCIAS}

1. Vecina Neto, G. Gestão de Recursos Materiais e de Medicamentos, volume 12 [Internet]. São Paulo: Faculdade de Saúde Pública da Universidade de São Paulo, 1998 [acesso em: 20 set. 2017]. Disponível em:

http://andromeda.ensp.fiocruz.br/visa/files/Volume12.pdf.

2. Castro LC, Castilho V. The cost of waste of consumable materials in a surgical center. Rev Lat Am Enfermagem [Internet]. 2013 [acesso em: 20 set. 2017];21(6):1228-34. Disponível em: http://dx.doi.org/10.1590/01041169.2920.2358.

3. World Health Organization. Health systems financing: the path to universal coverage. World health report 2010 [Internet]. Geneva: World Health Organization, 2010 [acesso em: 20 set. 2017]. Disponível em:

http://www.who.int/whr/2010/en/.

4. Ministério da Saúde, Secretaria Executiva. Ministério da Saúde e municípios: juntos pelo acesso integral e de qualidade à saúde [Internet]. 2ª ed. Brasília: Ministério da Saúde, 2013 [acesso em: 20 set. 2017]. Disponível em: http://bvsms.saude.gov.br/bvs/publicacoes/ministerio_municipios_juntos_acesso_integral.pdf.

5. Anhaia SA, Almeida SMO, Santos RF, Abrão FMS, Costa AM, Freitas CMS. Desafios na alocação de recursos em saúde: uma abordagem ética. Revista de enfermagem UFPE on line [Internet]. 2011 [acesso em: 20 set.

2017];5(3):836-42. Disponível em:

http://www.revista.ufpe.br/revistaenfermagem/index.php/revista/article/view/1352.

6. Zuliani LL, Jericó MC, Castro LC, Soler ZASG. Consumo e custo de recursos materiais em unidades pediátricas de terapia intensiva e semi-intensiva. Rev Bras Enferm [Internet]. 2012 [acesso em: 20 set. 2017];65(6):969-76.

Disponível em: http://dx.doi.org/10.1590/S0034-71672012000600013.

7. Bogo PC, Bernardino E, Castilho V, Cruz EDA. The nurse in the management of materials in teaching hospitals. Rev Esc Enferm USP [Internet]. 2015 [acesso em: 20 set. 2017];49(4):632-9. Disponível em:

http://dx.doi.org/10.1590/S0080-623420150000400014.

8. Castilho V, Castro LC, Couto AT, Maia FOM, Sasaki NY, Nomura FH et al. Levantamento das principais fontes de desperdício de unidades assistenciais de um hospital universitário. Rev Esc Enferm USP [Internet]. 2011 [acesso em: 20 set. 2017];45(esp.):1613-20. Disponível em: http://dx.doi.org/10.1590/S0080-62342011000700012.

9. Aranha GTC, Vieira RW. Estudo de um dos indicadores do custo de qualidade: o desperdício. RAS. Revista de administração em saúde [Internet]. 2004 [acesso em: 20 set. 2017];6(23):43-55. Disponível em:

http://www.ufjf.br/oliveira_junior/files/2011/08/ARANHA-e-VIEIRA_2004.pdf.

10. Lopes LA, Dyniewicz AM, Kalinowski LC. Gerenciamento de materiais e custos hospitalares em UTI neonatal. Cogitare Enferm [Internet]. 2010 [acesso em: 20 set. 2017];15(2):278-85. Disponível em:

http://dx.doi.org/10.5380/ce.v15i2.17862.

11. Goff SL, Kleppel R, Lindenauer PK, Rothberg MB. Hospital workers' perceptions of waste: a qualitative study involving photo-elicitation. BMJ Qual Saf [Internet]. 2013 [acesso em: 20 set. 2017];22(10):826-35. Disponível em: http://dx.doi.org/10.1136/bmjqs-2012-001683.

12. Pereira MS, Souza ACS. Problemas ambientais, sustentabilidade e a pesquisa em enfermagem. Rev. Eletr. Enf. [Internet]. 2013 [acesso em: 20 set. 2017];15(2):311-2. Disponível em: http://dx.doi.org/10.5216/ree.v15i2.15161. 13. Moreira LMV, Saes SO, Doria AB, Trenti FLC. Recursos materiais na enfermagem: um estudo sobre o conhecimento de custos sob a ótica dos alunos de graduação. Salusvitta [Internet]. 2002 [acesso em: 20 set. 2017];21(3):27-33. Disponível em: https://secure.usc.br/static/biblioteca/salusvita/salusvita_v21_n3_2002_art 01_por.pdf.

14. Beccaria LM, Pereira RAM, Torres JV, Capocianco TD, Barbosa TP, Poletti NAA. Custos de materiais hospitalares: percepção de docentes, acadêmicos e equipe de enfermagem. Revista de enfermagem UFPE on line [Internet]. 2013 [acesso em: 20 set. 2017];7(12):6834-40. Disponível em: http://www.revista.ufpe.br/revistaenfermagem/index.php/revista/article/view/4506. 
15. Universidade Federal de São Paulo, Pró-Reitoria de Graduação. Consulta Matriz Curricular do Curso [Internet]. São Paulo: UNIFESP, c2013-2017 [acesso em: 20 set. 2017]. Disponível em: https://www.unifesp.br/reitoria/prograd/proreitoria-de-graduacao/cursos/matriz-curricular.

16. Dallora MELV, Forster AC. A importância da gestão de custos em hospitais de ensino - considerações teóricas. Med (Ribeirão Preto Online) [Internet]. 2008 [acesso em: 20 set. 2017];41(2):135-42. Disponível em: http://dx.doi.org/10.11606/issn.2176-7262.v41i2p135-142.

17. Fugulin FMT, Lima AFC, Castilho V, Guimarães CP, Carvalho A, Gaidzinski RR. Quadro de profissionais de enfermagem em unidades médico-cirúrgicas de hospitais de ensino: composição e custos. Rev Esc Enferm USP [Internet]. 2015 [acesso em: 20 set. 2017];49(esp. 2):48-54. Disponível em: http://dx.doi.org/10.1590/S0080623420150000800007.

18. Sari V, Camponogara S. Challenges of environmental education in a hospital institution. Texto Context - Enferm [Internet]. 2014 [acesso em: 20 set. 2017];23(2):469-78. Disponível em: http://dx.doi.org/10.1590/010407072014001130013.

19. Peres RR, Camponogara S, Costa VZ, Terra MG, Nietsche EA. Educação ambiental para docentes enfermeiros: percepção e relação com a formação do enfermeiro. Rev Gaucha Enferm [Internet]. 2015 [acesso em: 20 set. 2017];36(esp.):85-93. Disponível em: http://dx.doi.org/10.1590/1983-1447.2015.esp.56696.

20. Oliveira WT, Rodrigues AVD, Haddad MCL, Vannuch MTO, Taldivo MA. Concepções de enfermeiros de um hospital universitário público sobre o relatório gerencial de custos. Rev Esc Enferm USP [Internet]. 2012 [acesso em: 20 set. 2017];46(5):1184-91. Disponível em: http://dx.doi.org/10.1590/\$0080-62342012000500021.

21. Francisco IMF, Castilho V. O ensino de custos nas escolas de graduação em enfermagem. Rev Esc Enferm USP [Internet]. 2004 [acesso em: 20 set. 2017];38(3):317-25. Disponível em: http://dx.doi.org/10.1590/S008062342004000300010.

22. Meira MDD, Kurgant P. Educação em enfermagem: avaliação da formação por egressos, empregadores e docentes. Rev Bras Enferm [Internet]. 2016 [acesso em: 20 set. 2017];69(1):10-5. Disponível em:

http://dx.doi.org/10.1590/0034-7167.2016690102i.

23. Lourenço KG, Castilho V. Classificação $A B C$ dos materiais: uma ferramenta gerencial de custos em enfermagem. Rev Bras Enferm [Internet]. 2006 [acesso em: 20 set. 2017];59(1):52-5. Disponível em: http://dx.doi.org/10.1590/S0034-71672006000100010.

24. Basak T, Unver V, Moss J, Watts P, Gaioso V. Beginning and advanced students' perceptions of the use of low- and high-fidelity mannequins in nursing simulation. Nurse Educ Today [Internet]. 2016 [acesso em: 20 set. 2017];36:37-43. Disponível em: http://dx.doi.org/10.1016/j.nedt.2015.07.020.

25. Valadares AFM, Magro MCS. Opinião dos estudantes de enfermagem sobre a simulação realística e o estágio curricular em cenário hospitalar. Acta Paul Enferm [Internet]. 2014 [acesso em: 20 set. 2017];27(2):138-43. Disponível em: http://dx.doi.org/10.1590/1982-0194201400025. 